\title{
Validation of a particle tracking analysis method for the size determination of nano- and microparticles
}

\author{
Vikram Kestens (D) - Vassili Bozatzidis • \\ Pieter-Jan De Temmerman • Yannic Ramaye • \\ Gert Roebben
}

Received: 23 May 2017 / Accepted: 13 July 2017 / Published online: 4 August 2017

(C) The Author(s) 2017. This article is an open access publication

\begin{abstract}
Particle tracking analysis (PTA) is an emerging technique suitable for size analysis of particles with external dimensions in the nano- and sub-micrometre scale range. Only limited attempts have so far been made to investigate and quantify the performance of the PTA method for particle size analysis. This article presents the results of a validation study during which selected colloidal silica and polystyrene latex reference materials with particle sizes in the range of $20 \mathrm{~nm}$ to $200 \mathrm{~nm}$ were analysed with NS500 and LM10-HSBF NanoSight instruments and video analysis software NTA 2.3 and NTA 3.0. Key performance characteristics such as working range, linearity, limit of detection, limit of quantification, sensitivity, robustness, precision and trueness were examined according to recommendations proposed by EURACHEM. A model for measurement uncertainty estimation following the principles described in ISO/IEC Guide 98-3 was used for quantifying random and systematic variations. For nominal $50 \mathrm{~nm}$
\end{abstract}

Electronic supplementary material The online version of this article (doi:10.1007/s11051-017-3966-8) contains supplementary material, which is available to authorized users.

V. Kestens $(\triangle) \cdot$ V. Bozatzidis $\cdot$ Y. Ramaye $\cdot$ G. Roebben Joint Research Centre (JRC), Directorate Health, Consumers and Reference Materials, European Commission, Retieseweg 111, 2440 Geel, Belgium

e-mail: vikram.kestens@ec.europa.eu

P.-J. De Temmerman

Service Trace Elements and Nanomaterials, Veterinary and Agrochemical Research Centre (CODA-CERVA), Groeselenberg 99, 1180 Brussels, Belgium and $100 \mathrm{~nm}$ polystyrene and a nominal $80 \mathrm{~nm}$ silica reference materials, the relative expanded measurement uncertainties for the three measurands of interest, being the mode, median and arithmetic mean of the numberweighted particle size distribution, varied from about $10 \%$ to $12 \%$. For the nominal $50 \mathrm{~nm}$ polystyrene material, the relative expanded uncertainty of the arithmetic mean of the particle size distributions increased up to $18 \%$ which was due to the presence of agglomerates. Data analysis was performed with software NTA 2.3 and NTA 3.0. The latter showed to be superior in terms of sensitivity and resolution.

Keywords Measurement uncertainty · Method validation $\cdot$ Nanoparticles $\cdot$ Particle size analysis $\cdot$ Particle tracking analysis $\cdot$ Reference material

\section{Introduction}

Achieving accurate particle size results is critical for many different industrial and research applications and processes. For nanoparticles, this demand has become even more important with the adoption of the Recommendation 'on the definition of a nanomaterial' (EC 2011) which is based on particle number-based distributions of particle size values. A wide variety of techniques such as scanning and transmission electron microscopy (SEM and TEM), dynamic light scattering (DLS), centrifugal liquid sedimentation (CLS), scanning probe microscopy (SPM) and small-angle X-ray scattering (SAXS) 
are available for particle characterisation. Particularly in the context of the EC nanomaterial definition, SEM and TEM have attracted most attention as the obtained results are intrinsically number-weighted. The main disadvantages of these electron microscopy-based techniques are the low sample throughput, the poor statistical sampling that results from the relatively low number of particles counted by the analysis, high measurement time and cost, and the high level of operator expertise needed.

Particle tracking analysis (PTA), often referred to as nanoparticle tracking analysis (NTA), is a technique (Malloy and Carr 2006) that intends to overcome several of the limitations mentioned above, i.e. for a single measurement, a significantly higher number of particles can be analysed in typically less than $10 \mathrm{~min}$. As a result, the technique is sometimes promoted as a costeffective alternative to SEM and TEM analyses. In contrast to the ensemble-based DLS technique, PTA measurements are performed on a particle-by-particle basis and are therefore assumed to be less prone to interference caused by agglomerates or larger particles when present in a heterogeneous test sample.

PTA combines laser light scattering with an optical microscope. The microscope unit is mounted almost perpendicular to the direction of the incident laser beam and is equipped with a camera which records the light scattered by the individual particles that are undergoing Brownian motion. The instrument software tracks the trajectories of the particles detected in the microscope's field of view and determines their mean-square displacements in two dimensions (Qian et al. 1991). The translational diffusion coefficient of each tracked particle is then computed and converted into a sphere-equivalent hydrodynamic diameter according to the following modified Stokes-Einstein Eq. (1):

$\overline{\frac{(x, y)^{2}}{4 t}}=D_{x, y}=\frac{k_{\mathrm{B}} T}{3 \pi \eta d}$

where $\overline{(x, y)^{2}}$ is the mean-square displacement in two dimensions, $D_{x, y}$ is the translational diffusion coefficient in two dimensions, $k_{\mathrm{B}}$ is Boltzmann's constant, $T$ is the sample temperature, $t$ is the time, $\eta$ is the viscosity of the dispersion medium and $d$ is the sphere-equivalent hydrodynamic diameter.

Particular attention must be paid to the fact that the validity of the measurement model, as presented in Eq. (1), is based on a number of assumptions: (1) all particles must be freely and uncorrelated moving in all three dimensions under the influence of Brownian diffusion and (2) particles must be colloidally stable during the measurement time, $t$. In addition, particles with a non-spherical or non-equiaxial shape are subjected to a combination of translational and rotational diffusion. In particular for high-aspect ratio particles (e.g. carbon nanotubes, rod- and plate-like particles), rotational movement causes flickering of the scattered light and this can perturb the tracking and detection of the particles and, as a result, significantly affect the statistical reliability of the measured equivalent hydrodynamic diameter results (Gallego-Urrea et al. 2014). The data analysis algorithms implemented in the particle tracking software which have been used in the presented work do not account for rotational diffusion.

The PTA measurement procedure generally consists of sample preparation, video recording and video analysis. Sample preparation often requires diluting the supplied sample in an aqueous medium such as purified water until the particle concentration is within the recommended range of $10^{7}$ to $10^{9}$ particles $/ \mathrm{mL}$. According to Krueger et al. (2016), the process that covers the recording and analysis of videos can be prone to bias as various acquisition and analysis parameters must be optimised and defined by the individual analyst. The adjustment of these parameters can significantly impact the shape of the measured size distribution (Filipe et al. 2010). The key parameters for data collection are the gain and the shutter speed of the camera, the capture duration, and the alignment of the sample holder and the optical system, while the main critical parameters for data analysis are the screen gain, detection threshold, the minimum expected particle size and blur (Patois et al. 2012). De Temmerman et al. (2014a) systematically compared PTA results with results from TEM and estimated measurement uncertainties for both sets of data. The reported PTA relative expanded measurement uncertainties were $7 \%, 9 \%$ and $14 \%$ for nominal $200 \mathrm{~nm}$ and $100 \mathrm{~nm}$ polystyrene and $30 \mathrm{~nm}$ gold, respectively. These uncertainties only cover measurement variation due to method repeatability and intermediate precision. Sauvain et al. (2017) recently validated a PTA method for measuring the size and number concentration of particles in a biological medium (i.e. exhaled breath condensate). In their validation study, measurements were also performed on nominal $100 \mathrm{~nm}$ PSL particles dispersed in pure water and in biological medium. The relative standard uncertainty for precision was estimated 
as $4.5 \%$ (in water) and $8 \%$ (in biological medium). However, both De Temmerman et al. and Sauvain et al. did not use certified reference materials for quantifying the aspect of method trueness. No other attempts have been made so far to fully validate the PTA method for particle size analysis using a variety of non-certified and certified reference materials and according to recommendations proposed by EURACHEM (2014).

This study aims to demonstrate, through a validation study, that the PTA method is fit for the purpose of determining selected characteristic values of the number-weighted particle size distributions (PSD) of populations of monomodal and bimodal mixtures of silica and polystyrene particles in aqueous suspension. Silica and polystyrene suspensions were selected as model samples as the particles have a (near-)spherical shape, which is an assumption for the validity of the Stokes-Einstein equation, and because they are available as reference materials in a variety of sizes. Reference materials come with a quantified level of heterogeneity, and this reduces the risk that random variation is interpreted as a method characteristic while in practice it is due to sample heterogeneity. For the three targeted measurands (i.e. mode, median and arithmetic mean of a number-weighted PSD), relative expanded uncertainties up to a maximum of about $10 \%$ would be acceptable. Measurement uncertainties in the range of $3 \%$ to $16 \%$ have been frequently reported for the size results of nanoparticles measured by other techniques such as transmission electron microscopy (De Temmerman et al. 2014a), dynamic light scattering and centrifugal liquid sedimentation (Braun et al. 2011). This study also provides a systematic comparison between results computed by the previous, but still commonly used, software version NTA 2.3 and one of the newer software versions NTA 3.0, which employs an improved data fitting algorithm.

\section{Materials and methods}

\section{PTA instrument}

Optimisation and validation experiments were carried out with NanoSight instruments models NS500 and LM10HSBF (Malvern Instruments Ltd., Worcestershire, UK). Both PTA instruments are equipped with an optical microscope objective lens of 20x magnification, a laser diode with a wavelength of $405 \mathrm{~nm}$ and maximum power output of $65 \mathrm{~mW}$ and an Orca-Flash 2.8 scientific complementary metal-oxide-semiconductor (sCMOS) camera (Hamamatsu Photonics, Hamamatsu City, Japan). The sCMOS cameras were operated at 25 frames per second. The field of view of the optical microscopes was calibrated by the manufacturer against the $100 \mathrm{~nm}$ size value (as indicated by TEM) of a polystyrene particle standard (Thermo Scientific, Fremont, USA).

The LM10-HSBF device was used for measuring ERM-FD101b during the trueness assessment stage. All other measurements were performed with the NS500 device. Particle size results were calculated from the determined translational diffusion coefficients using a modified version of the Stokes-Einstein formula, Eq. (1) (Filipe et al. 2010). The viscosity of the suspension medium, which was assumed to be that of pure water, was for each analysis automatically adjusted according to the measured temperature. Video files were acquired using the instrument software version NTA 2.3 (Build 33) and were analysed with both software versions NTA 2.3 (Build 33) and NTA 3.0 (Build 69). Apart from the redesigned interface and integrated hardware detection and communication, NTA 3.0 employs an improved and high-resolution finite track length adjustment (FTLA) particle size distribution algorithm which provides a better separation of different particle size populations (Walker 2012). Compared to the algorithm used in NTA 2.3 and earlier versions, the FTLA algorithm (algorithm described by Walker (2012) with some proprietary modifications) is model independent in that there is no assumption that the sample is monomodal. The latest software versions currently available are NTA 3.1 and NTA 3.2. The performance of these software versions was not investigated as they were released by the manufacturer after our validation study. NTA 3.1 and NTA 3.2 use the same FTLA data analysis algorithms as the one implemented in NTA 3.0. Hence, it can be assumed that the measurement uncertainties estimated from the validation data will also be valid for particle size results analysed with NTA 3.1 and NTA 3.2.

\section{Test materials}

Three colloidal silica and ten polystyrene latex (PSL) reference materials were analysed during the optimisation and validation studies. An overview of the different materials and relevant assigned particle size values extracted from the material certificates is given in Table 1. 


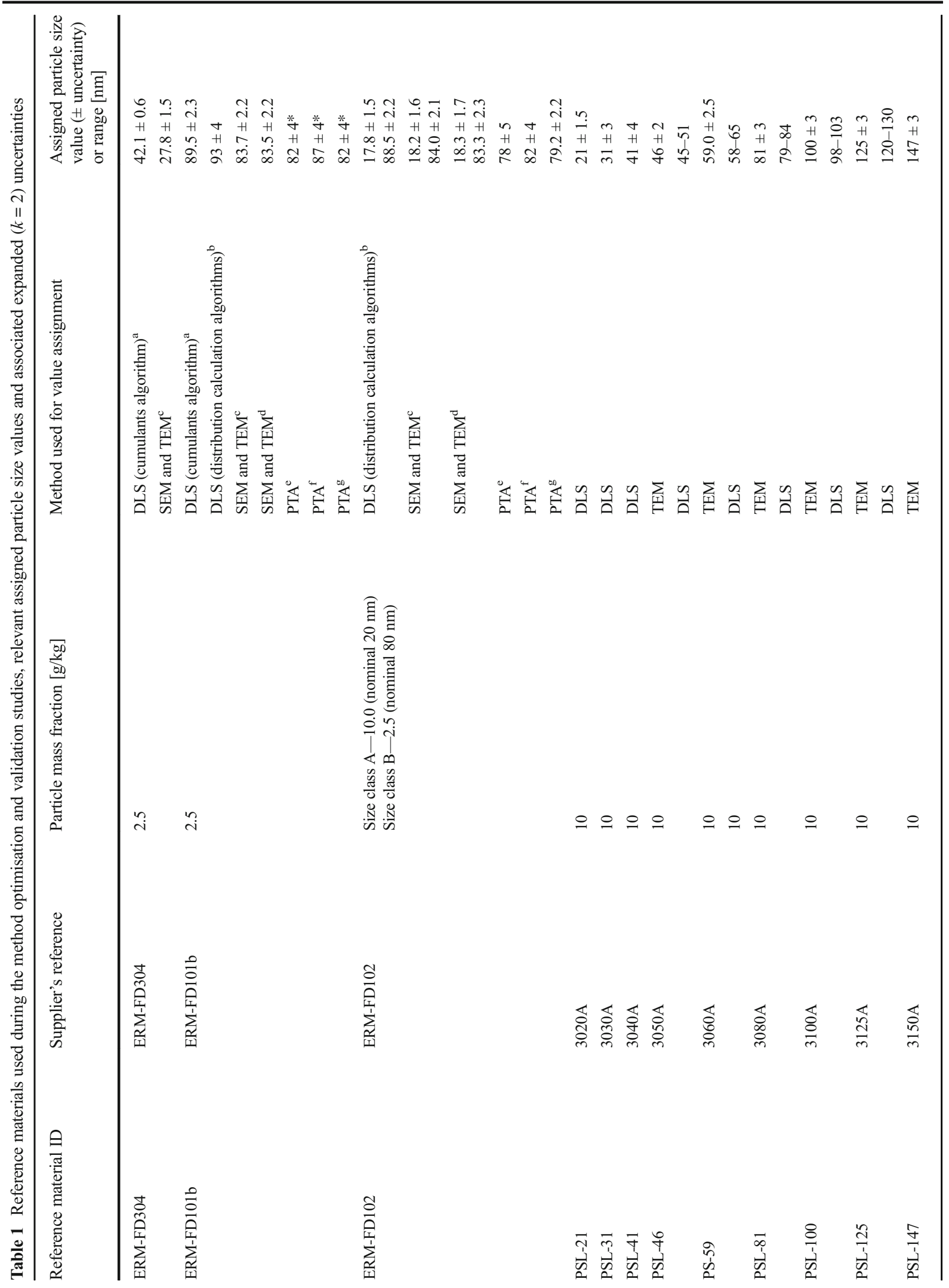




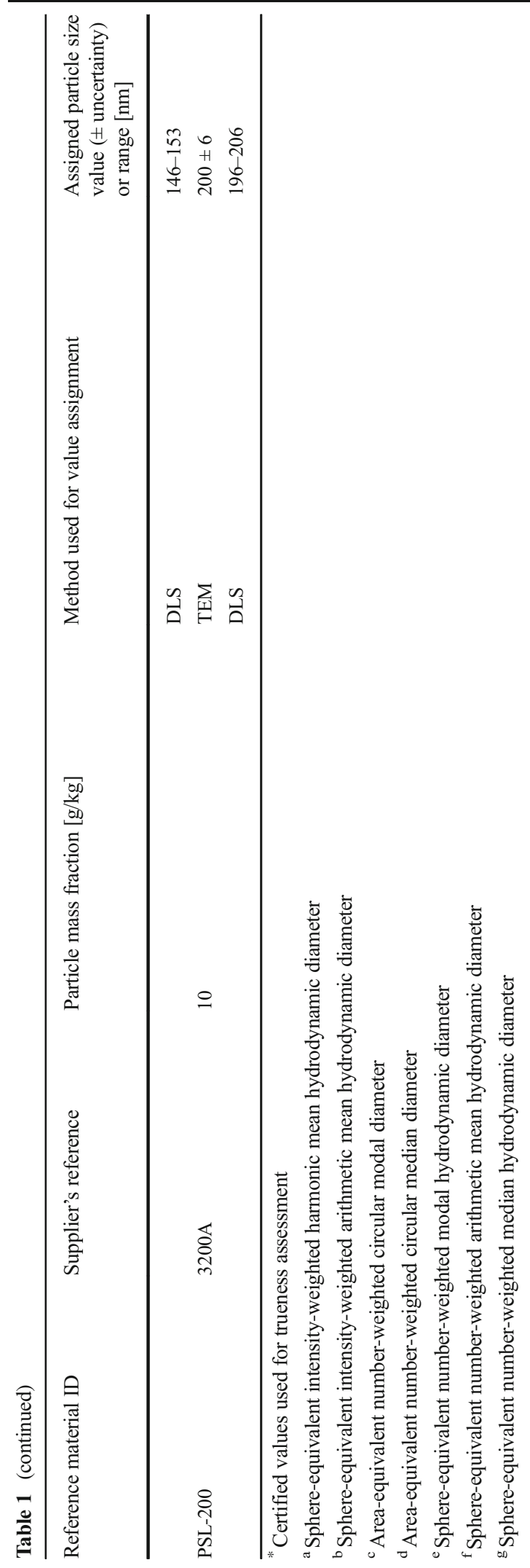

The colloidal silica reference materials were provided by the Directorate-General Joint Research Centre (JRC) of the European Commission (Geel, Belgium). While ERM-FD304 and ERM-FD101b have a monomodal PSD, the PSD of ERM-FD102 has two main modes which are further referred to as size class $A$ and size class B. ERM-FD102 also contains a third minor fraction of particles with diameters in the range of $40 \mathrm{~nm}$ to $50 \mathrm{~nm}$. However, the distribution characteristics of this population of particles were not assigned. All three colloidal silica materials are certified reference materials, but only ERM-FD101b comes with certified values for the mode, median and arithmetic mean of the particle number-weighted size distributions obtained by PTA. Hence, only PTA-certified values assigned to ERM-FD101b were used for assessing the trueness of the validated PTA method. ERM-FD304 and ERMFD102 are certified reference materials too because some of the assigned values have been obtained with validated methods (e.g. DLS, SEM, TEM). However, for ERM-FD304, no particle size value has been assigned for PTA. For ERM-FD102, the assigned indicative values for PTA originate from only three laboratories. A minimum of six laboratories is usually required for the assignment of a certified value. Therefore, in this validation study, ERM-FD304 and ERM-FD102 were regarded and used as (non-certified) reference materials.

The monomodal PSL materials with assigned diameters ranging from $21 \mathrm{~nm}$ to $200 \mathrm{~nm}$ were purchased from Thermo Scientific (Fremont, USA). This size range was particularly chosen as it fairly matches the size range of manufactured nanoparticles used in food, feed and cosmetic products (Contado 2015; Peters et al. 2016). To evaluate the relative resolution, different bimodal mixtures of different peak ratios were prepared from selected monodisperse PSL materials (Table 2). The nominal particle number concentration was calculated from the theoretical mass fraction $(10 \mathrm{~g} / \mathrm{kg})$, the particle density $\left(1.05 \mathrm{~g} / \mathrm{cm}^{3}\right)$ and the assigned particle diameter.

Sample preparation

The as-received reference materials were diluted in purified water. The purified water was prepared from regular tap water that had subsequently undergone reverse osmosis, UV-irradiation and filtration through a membrane with nominal pore sizes of $220 \mathrm{~nm}$ (Merck Millipore Inc., Billerica, MA, USA). The purified water, which had a resistivity of $18.2 \mathrm{M} \Omega \mathrm{cm}$ at $25^{\circ} \mathrm{C}$, was 
Table 2 Specifications of the bimodal PSL mixtures

\begin{tabular}{|c|c|c|c|c|}
\hline \multirow[t]{2}{*}{ Test material ID } & \multicolumn{2}{|c|}{ Monodisperse PSL materials } & \multicolumn{2}{|c|}{ Nominal particle number concentration $\left[\times 10^{7}\right.$ particles $\left./ \mathrm{mL}\right]$} \\
\hline & Fraction 1 & Fraction 2 & Fraction 1 & Fraction 2 \\
\hline PSL-50-100_2:1 & PSL-50 & PSL-100 & 7.6 & 3.8 \\
\hline PSL-50-100_4:1 & PSL-50 & PSL-100 & 15.3 & 3.8 \\
\hline PSL-60-100_1.2:1 & PSL-60 & PSL-100 & 4.4 & 3.8 \\
\hline PSL-60-100_2.3:1 & PSL-60 & PSL-100 & 8.8 & 3.8 \\
\hline PSL-50-80_3.6:1 & PSL-50 & PSL-80 & 135.0 & 37.3 \\
\hline PSL-80-100_3.1:1 & PSL-80 & PSL-100 & 37.3 & 11.9 \\
\hline PSL-50-60_1.7:1 & PSL-50 & PSL-60 & 30.6 & 17.7 \\
\hline PSL-50-60_5.2:1 & PSL-50 & PSL-60 & 91.8 & 17.7 \\
\hline
\end{tabular}

additionally passed through a membrane filter with nominal pore sizes of $100 \mathrm{~nm}$ to remove particles that were not retained by the $220 \mathrm{~nm}$ pore size filter of the purification system. Before each series of measurements, the cleanliness of the purified water was checked at the required instrument settings to ensure that the concentration of particles present in the purified water was below the limit of detection.

\section{Results and discussion}

The method validation study and measurement uncertainty estimations were carried out according to the EURACHEM (2014) and ISO/IEC Guide 98-3 (2008) guidelines. The method performance characteristics which were considered to be relevant for the PTA method were selectivity, calibration/working range, limit of detection (LOD), limit of quantification (LOQ), sensitivity, robustness and accuracy (i.e. precision and trueness). The measurands of interest were the mode, median and arithmetic mean of the particle numberweighted distribution of sphere-equivalent hydrodynamic particle diameter values.

\section{Selectivity}

Selectivity relates to the extent to which a method can be used to determine particular analytes in mixtures or matrices without significant interferences from other components of similar behaviour.

In this study, all test samples were diluted and dispersed in purified water. Due to their difference in refractive index and their very small size, water molecules scatter light much less than silica and polystyrene particles, and it can, therefore, be assumed that the water as background does not significantly interfere with the light scattered by the dispersed particles.

For heterogeneous samples, the PTA method is not selective because the instrument detects all scattered light regardless of the kind of particle that is the scattering source. Agglomerates and other undesired large particles can scatter light more intensively and can be the cause of interferences, i.e. the clouding of underlying small particles, and can drastically perturb the PSD.

\section{Calibration, working range, LOD and LOQ}

The PTA working range (and LOD and LOQ) depends both on particle size and particle concentration. According to the instrument manufacturer, the PTA method can measure the size of particles with diameters of $10 \mathrm{~nm}$ to $2 \mu \mathrm{m}$ and particle concentrations in the range of $10^{7}$ to $10^{9}$ particles $/ \mathrm{mL}$. The influence of the particle concentration on the determination of the mean, median and modal values of the PSDs of PSL-100 is discussed and shown in the Electronic supplementary information (ESI). These results show that within a particle concentration range of $1.9 \times 10^{6}$ to $1.9 \times 10^{9}$ particles $/ \mathrm{mL}$, both the NTA 2.3 and NTA 3.0 software calculate mean and modal particle size results that agree with the assigned reference value within $\pm 1.5 \%$ (relative standard uncertainty).

The actual lower limit of the working range for particle size depends on the light scattering properties (e.g. refractive index contrast) of the particles and the type of camera used. On the other hand, the upper limit of the range is defined by the effective density of the 
particles which determines whether during the measurement the particles remain in suspension due to Brownian motion or whether sedimentation forces are dominant. For the presented PTA method, the working range for particle size measurements was confirmed and fixed based on the modal values of the particle numberweighted size distributions obtained for the reference materials listed in Table 1.

Particle sizing with PTA is an absolute method; in other words, it relies only on the first principles captured in Eq. (1). As a result, there is no need for the analyst to calibrate the instrument's signal response with particle size calibrants. However, the distances travelled by the particles and measured by the instrument software need to be correctly measured, and for this, a calibration constant has been determined by the manufacturer. PSL particles of $100 \mathrm{~nm}$ in diameter or an optical diffraction grid is typically used by the manufacturer to calibrate the length scale of the optical microscope's field of view and to convert pixels into the SI unit of length (metre). The instrument manufacturer states that in the case of the calibration grid, a distance of $100 \mu \mathrm{m}$ representing about 600 pixels is measured, and the userto-user variation is about 2 pixels or $0.4 \%$ at the extreme. No further details are available from the manufacturer concerning the calibration with the $100 \mathrm{~nm}$ PSL particles.

As can be seen from Fig. 1, measurements on eight different PSL particle size standards indicate that the one-point calibration yields a linear relationship $\left(R^{2}=0.999\right)$ between the means of the modal diameter results measured with software NTA 2.3 and the particle diameter values assigned to the tested PSL materials. The PSDs obtained for the smallest PSL (PSL-21) were multimodal, and the peak that corresponded to the individual nonagglomerated particles could not always be unambiguously determined due to the method's limit of quantification. Therefore, the linear fit does not include the results of PSL-21. The results obtained on the other PSL materials confirm the working range for monodisperse PSL particles with diameters between $31 \mathrm{~nm}$ and $200 \mathrm{~nm}$. The relative standard deviations (RSD) that were calculated for each set of nine replicate results varied between $2.4 \%$ (PSL81 ) and $7.3 \%$ (PSL-147). A significantly higher RSD of $13.1 \%$ was calculated for the results obtained for PSL-31. Since all measurement results were achieved under intermediate precision conditions, the RSD values give a first estimate of the precision of the PTA method. Considering the target relative expanded $(k=2$, confidence level of about 95\%) uncertainty of $10 \%$ (which is equivalent to a relative standard uncertainty of $5 \%$ at a confidence level of $68 \%$ ), the maximum relative standard uncertainty for precision should ideally be in the range of about $3 \%$ to $4 \%$. For PSL-31, the RSD value of $13 \%$ is significantly higher than $4 \%$, while the RSD values of the other materials vary from about $2 \%$ to $7 \%$. It must be stressed that these measurements were not carried out according to a nested study design and that possible sample-to-sample heterogeneity may also be included in the variances. Also, no trends between the RSD and particle size values were observed. Hence, we regard the lower limit $(31 \mathrm{~nm})$ of the range of the linear fit as the lower LOD and $41 \mathrm{~nm}$ as the lower LOQ. For PSL-200, an RSD value of $5.3 \%$ was calculated, fixing both the upper LOD and upper LOQ at $200 \mathrm{~nm}$.

The second set of data presented in Fig. 1 shows the absolute difference (i.e. bias) between the mean measured modal values and the theoretical values and their associated expanded uncertainties. For the tested PSL materials, the PTA size values were systematically higher than the size values that were assigned by the manufacturer using TEM and DLS. This bias may be caused by a combination of different factors. On the one hand, differences between particle size results can stem also from the different physical principles and data analysis algorithms of the measurement techniques (Kestens et al. 2016). On the other hand, as highlighted by others, polymeric particles can shrink from prolonged exposure to the focussed electron beams of electron microscopes (McDonald et al. 1977).

At this stage, only two colloidal silica materials were tested: ERM-FD304 and ERM-FD102. As can be seen from the large error bars (or shaded areas) in Fig. 2, ERM-FD304 could not be analysed with an acceptable degree of precision. Also, the modal values of the PSDs were significantly larger than the certified (42.1 nm for DLS) and indicative $(27.8 \mathrm{~nm}$ for electron microscopy) values. Similar observations were recently made by Tuoriniemi et al. (2014) and Nicolet et al. (2016) who reported biased PTA results of about $67 \mathrm{~nm}$ and $87 \mathrm{~nm}$ for silica nanoparticles of nominally $35 \mathrm{~nm}$ and $48 \mathrm{~nm}$ in diameter, respectively. Nicolet et al. suggested that the systematic error was due to the 
Fig. 1 Results of linear relation between assigned and measured particle size (squares; left y-axis); bias (circles with error bars indicating standard deviations; right $y$-axis) between the mean measured modal particle diameter and particle diameter assigned by TEM or DLS (in the case of PSL-

21, PSL-31 and PSL-41).

Expanded uncertainties associated with the assigned values are indicated with en dash symbols (-)

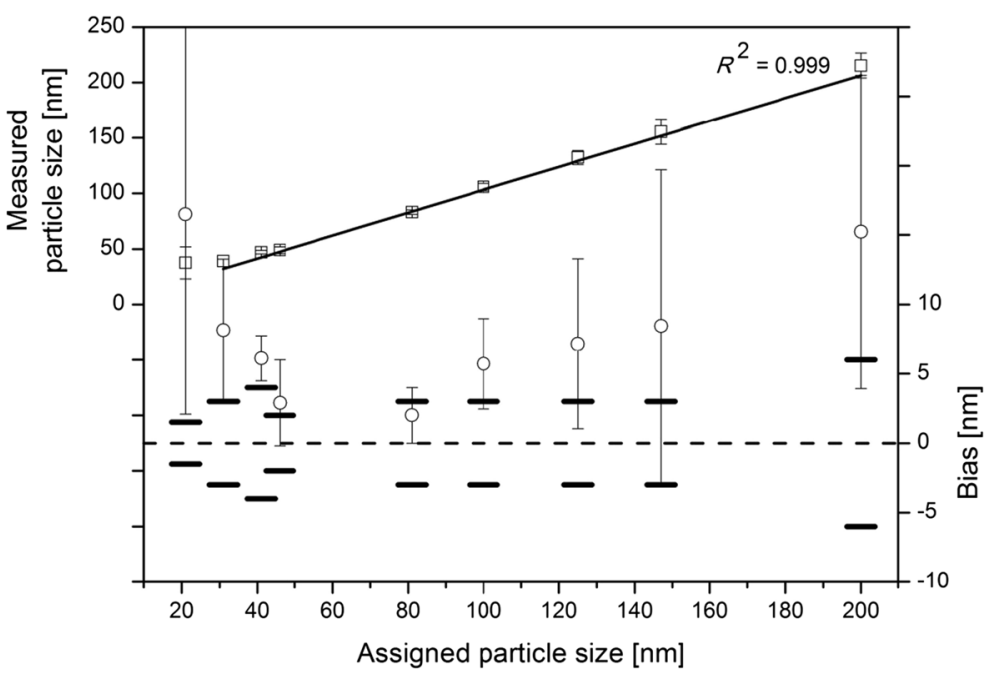

method's insensitivity to the small particles in the sample and the counting of particle clusters instead. Another possible explanation for the bias is that the laser beam exhibits a Gaussian intensity profile when illuminating the sample. Large and strongly scattering particles at the less intense beam edge may remain detectable and quantifiable, while the scattered light of small particles with a low refractive index contrast may drop below the LOQ (Malloy and Carr 2006). An acceptable degree of precision was obtained for the results that were associated with the peak of the size class B fraction of ERM-FD102 (Fig. 3). The size class A fraction could hardly be detected (see insert in Fig. 3). Only for few PSDs, a very small peak between $25 \mathrm{~nm}$ and $30 \mathrm{~nm}$, which may present the nominal $20 \mathrm{~nm}$ particles, could be observed. The third minor particle population could be seen for practically all PSDs in the size range of about $35 \mathrm{~nm}$ to $55 \mathrm{~nm}$. The mode of that fraction could, however, not be determined accurately. For silica particles, the lower LOD and LOQ can be concluded as about $40 \mathrm{~nm}$ (from ERM-FD304) and about $80 \mathrm{~nm}$ (from ERM-FD102), respectively. In agreement with the approaches used by Tsai et al. (2011) and De Temmerman et al. (2014a), this lower LOD agrees well with a theoretical LOD of about $33 \mathrm{~nm}$ which is calculated by comparing the
Fig. 2 Mean number-weighted PSD representing of ERMFD304 obtained by PTA. Shaded areas correspond to the standard deviation of 56 analyses of the same material

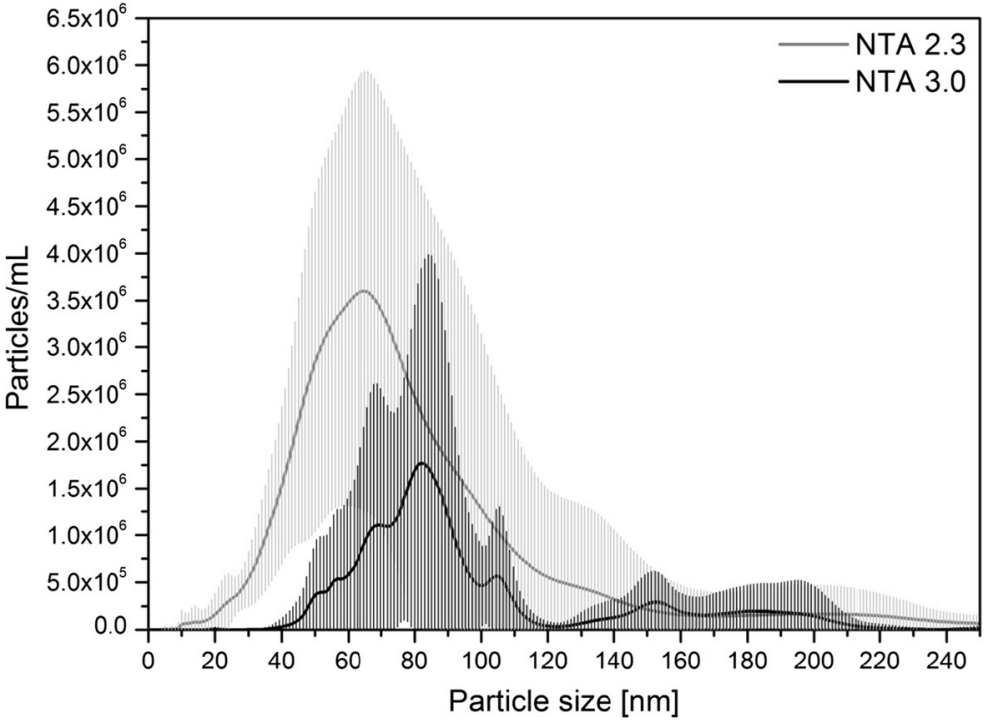


Fig. 3 Mean number-weighted PSD of ERM-FD102 obtained by PTA. Shaded areas correspond to the standard deviation of 54 analyses of the same material. The inset figure shows a zoomed region of the sub-70 $\mathrm{nm}$ particle size range indicating the presence of the third minor particle population (error bars are omitted for clarity)

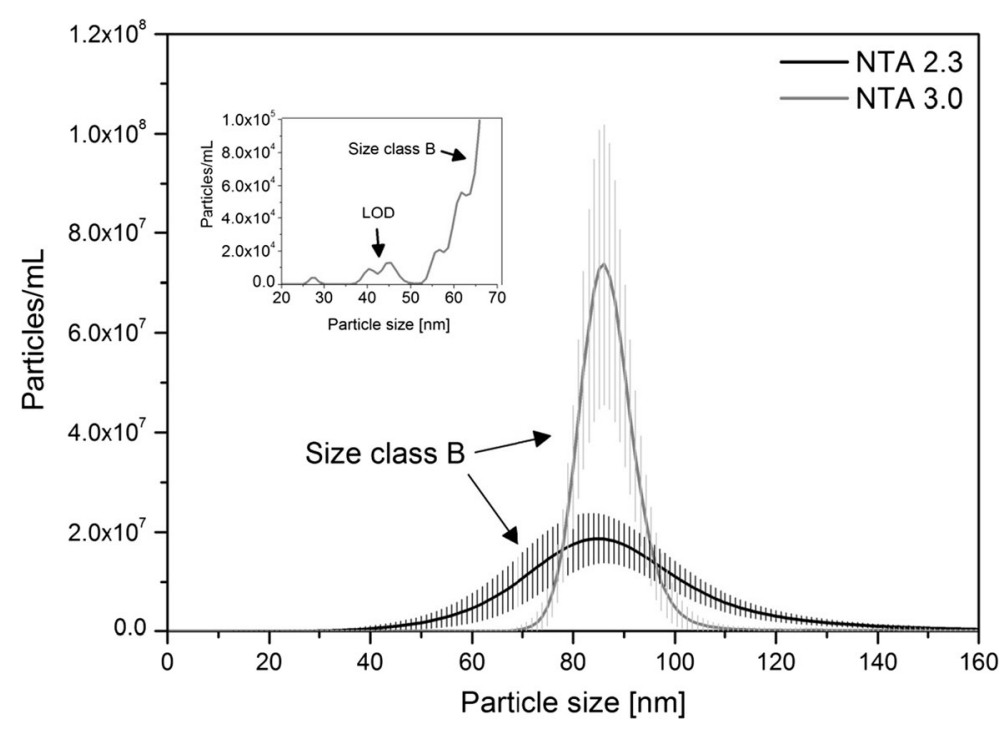

scattered light intensity ratios for polystyrene particles of $31 \mathrm{~nm}$ in diameter and a refractive index of 1.59 , and silica particles with a refractive index of 1.54.

\section{Sensitivity and resolution}

The sensitivity of the PTA method to detect and quantify, within its working range, different particle populations was investigated by preparing different bimodal mixtures of monodisperse PSL materials. Here, the sensitivity is quantitatively expressed in terms of resolution $\left(R_{\mathrm{S}}\right)$, which is a useful measure of the effectiveness of separation of two neighbouring peaks. An overview of the different valid approaches for calculating $R_{\mathrm{S}}$ values is provided in the ESI. The $R_{\mathrm{s}}$ values listed in Table 3 were calculated according to the resolution function which is given by Eq. S2. The necessary peak-related characteristic values such as the peak widths measured at half-height were estimated by fitting the experimental data of each peak, or the resolved parts thereof, with simulated monomodal data that followed a Gaussian function (Fig. S5).

Different method parameters can have an influence on the resolution. This study only compares the effect of the two different PSD algorithms used in software versions NTA 2.3 and NTA 3.0.

A complete peak separation could be obtained for PSL-50-100 and PSL-60-100 when using NTA 3.0

Table 3 Local maxima (mean \pm SD in $\mathrm{nm}$ ) of the bimodal peaks of the number-weighted PSDs of PSL bimodal mixtures and associated $R_{\mathrm{s}}$ values

\begin{tabular}{|c|c|c|c|c|c|c|c|}
\hline \multirow[t]{2}{*}{ Test material ID } & \multirow[t]{2}{*}{ Number of replicates } & \multicolumn{3}{|l|}{ NTA 2.3} & \multicolumn{3}{|l|}{ NTA 3.0} \\
\hline & & Fraction 1 & Fraction 2 & $R_{\mathrm{s}}$ & Fraction 1 & Fraction 2 & $R_{\mathrm{S}}$ \\
\hline PSL-50-100_2:1 & 3 & $55 \pm 3$ & $95 \pm 3$ & 0.7 & $57 \pm 5$ & $105 \pm 3$ & 2.0 \\
\hline PSL-50-100_4:1 & 3 & $51 \pm 3$ & $100 \pm 2$ & 0.9 & $53 \pm 2$ & $100 \pm 4$ & 1.9 \\
\hline PSL-60-100_1.2:1 & 4 & $62 \pm 5$ & $98 \pm 3$ & 0.7 & $65 \pm 3$ & $102 \pm 4$ & 1.4 \\
\hline PSL-60-100_2.3:1 & 4 & $63 \pm 2$ & $94 \pm 5$ & 0.5 & $65 \pm 4$ & $97 \pm 5$ & 1.2 \\
\hline PSL-50-80_3.6:1 & 9 & $<$ LOD & $78 \pm 2$ & N.A. & $53 \pm 13$ & $83 \pm 4$ & 1.0 \\
\hline PSL-80-100_3.1:1 & 3 & $86 \pm 3$ & $<\mathrm{LOD}$ & N.A. & $84 \pm 1$ & $100 \pm 2$ & 0.5 \\
\hline PSL-50-60_1.7:1 & 3 & $<\mathrm{LOD}$ & $59 \pm 2$ & N.A. & $<$ LOD & $63 \pm 1$ & N.A. \\
\hline PSL-50-60_5.2:1 & 3 & $48 \pm 1$ & $<\mathrm{LOD}$ & N.A. & $52^{\mathrm{a}}$ & $61^{\mathrm{a}}$ & $0.5^{\mathrm{a}}$ \\
\hline
\end{tabular}

N.A. not applicable as only one fraction could be detected

${ }^{\mathrm{a}}$ Based on a single measurement result 

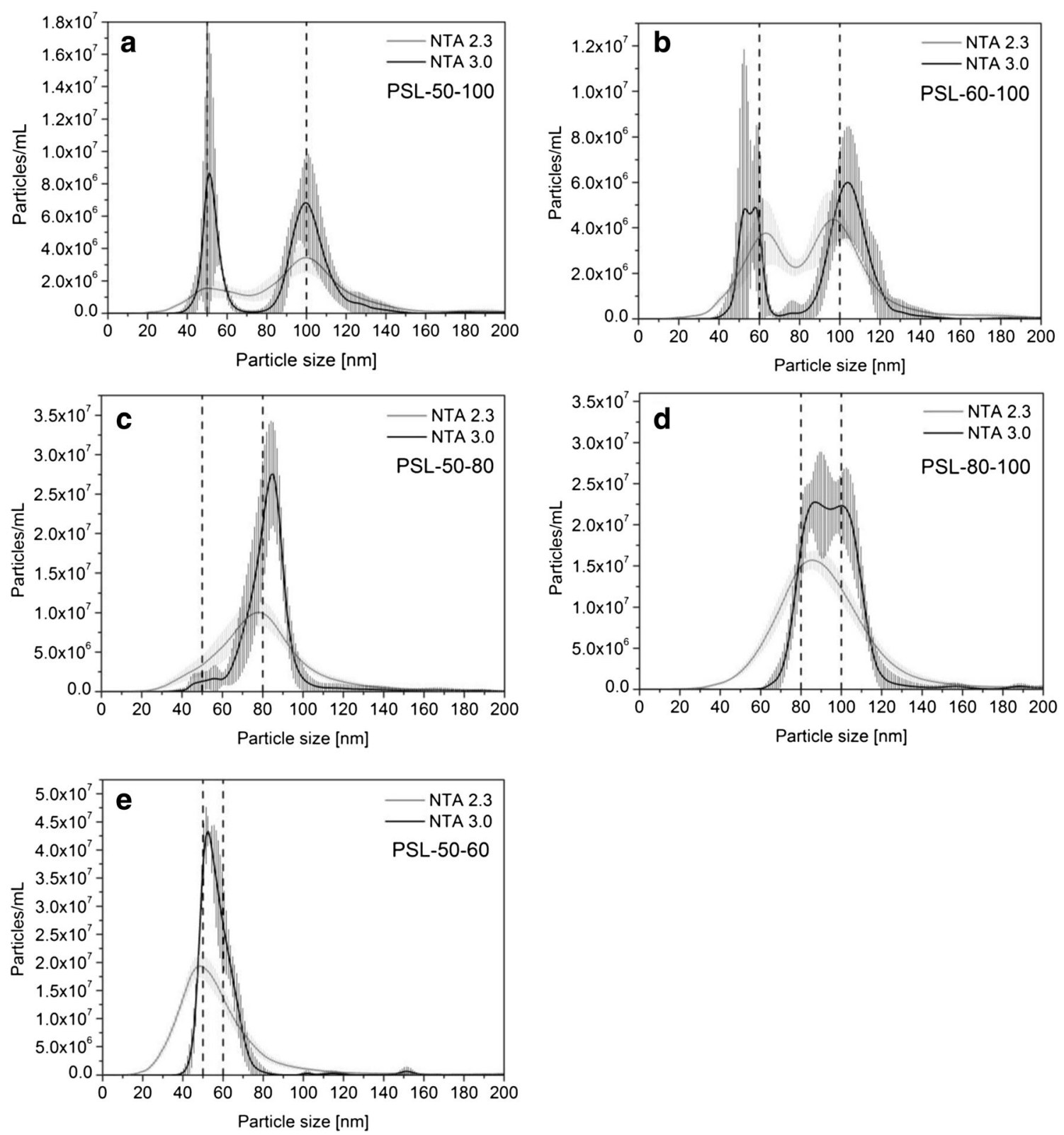

Fig. 4 Mean number-weighted PSDs of bimodal PSL mixtures obtained by PTA. Shaded areas correspond to the standard deviation of four analyses of the same sample, vertical dashed lines indicate the nominal particle size value of each population

while NTA 2.3 only achieved marginal peak separations (Fig. 4a, b). For PSL-50-80, PSL-80-100 and PSL-5060 , NTA 2.3 could only determine monomodal PSDs of which the modes were shifted towards one of the two particle populations (Fig. 4c-e). NTA 3.0 could marginally separate the peaks of the PSL-50-80 and PSL-80100 particles, allowing reliable determination of the associated local maxima. For the PSL-50-60 material, only one of the three acquired PSDs showed the presence of the two particle populations.

In general, it can be concluded that thanks to the FTLA algorithm, software NTA 3.0 provides superior resolution compared to software NTA 2.3. $R_{\mathrm{S}}$ values of $\geq 1.2$ correspond to complete peak separation, and a 
Table 4 Characteristic values (mean \pm SD of aliquot means, in $\mathrm{nm}$ ) of monomodal number-weighted PSDs

\begin{tabular}{|c|c|c|c|c|c|c|c|c|}
\hline \multirow{2}{*}{$\begin{array}{l}\text { Peak } \\
\text { characteristic }\end{array}$} & \multicolumn{4}{|l|}{ NTA 2.3} & \multicolumn{4}{|l|}{ NTA 3.0} \\
\hline & PSL-46 & PSL-100 & ERM-FD101b & $\begin{array}{l}\text { ERM-FD102 (size } \\
\text { class B) }\end{array}$ & PSL-46 & PSL-100 & ERM-FD101b & $\begin{array}{l}\text { ERM-FD102 (size } \\
\text { class B) }\end{array}$ \\
\hline Mean & $63 \pm 7$ & $119 \pm 12$ & $88 \pm 1$ & $94 \pm 4$ & $60 \pm 7$ & $111 \pm 7$ & $84 \pm 1$ & $91 \pm 3$ \\
\hline Mode & $49 \pm 3$ & $106 \pm 4$ & $82 \pm 1$ & $85 \pm 3$ & $51 \pm 1$ & $106 \pm 4$ & $87 \pm 1$ & $86 \pm 1$ \\
\hline $\operatorname{Median}\left(d_{50}\right)$ & $54 \pm 4$ & $111 \pm 4$ & $84 \pm 1$ & $88 \pm 2$ & $51 \pm 2$ & $106 \pm 4$ & $78 \pm 1$ & $86 \pm 1$ \\
\hline$d_{10}$ & $37 \pm 4$ & $88 \pm 4$ & N.A. & $67 \pm 2$ & $44 \pm 2$ & $98 \pm 4$ & N.A. & $79 \pm 1$ \\
\hline$d_{90}$ & $97 \pm 17$ & $158 \pm 39$ & N.A. & $120 \pm 5$ & $79 \pm 25$ & $132 \pm 39$ & N.A. & $95 \pm 3$ \\
\hline Span & $1.1 \pm 0.2$ & $0.6 \pm 0.1$ & N.A. & $0.6 \pm<0.1$ & $0.7 \pm 0.4$ & $0.3 \pm 0.2$ & N.A. & $0.2 \pm<0.1$ \\
\hline Breadth & $34 \pm 12$ & $35 \pm 14$ & N.A. & $35 \pm 9$ & $29 \pm 18$ & $21 \pm 16$ & N.A. & $27 \pm 13$ \\
\hline
\end{tabular}

N.A. no data available

complete peak analysis can be performed using simple statistics. $R_{\mathrm{S}}$ values of $<1.2$ indicate marginal peak separation, and the partially resolved particle populations can only be approximatively characterised using the local maxima.

Precision, trueness and measurement uncertainty estimation

The accuracy of the PTA data (i.e. the combination of precision and trueness) was determined by measuring PSL-46, PSL-100, ERM-FD102 and ERMFD101b. The first three reference materials were used for assessing the precision of the method by performing measurements under repeatability and intermediate precision conditions according to a nested design; a total of 20 aliquots were analysed over the course of 5 days (four aliquots per day) by one operator using the NS500 device. The trueness of the method was investigated by analysing nine replicates of ERM-FD101b over the course of 3 days (three aliquots per day) by another operator using the LM10-HSBF instrument. Each single aliquot result is the mean of three results obtained on three sub-samples that were consecutively loaded into the measurement cell and analysed under repeatability conditions. The precision and trueness of the PTA method was quantified for the modal, median and arithmetic mean central values of the numberweighted PSDs. An overview of the obtained results is given in Table 4. In addition to the modal, mean and median $\left(d_{50}\right)$ values, the instrument software also computes cumulative particle size results at the 10th $\left(d_{10}\right)$ and 90th $\left(d_{90}\right)$ percentiles. The percentile results can be used for calculating the span (i.e. $\left.\left(d_{90}-d_{10}\right) / d_{50}\right)$, which is a dimensionless measure of the distribution width relative to the median. The breadth of the PSD curve around the mean is given by $1 \times$ standard deviation (SD). These additional results are only informative, i.e. the performance of the PTA method to quantify a distribution of particle size results was outside the scope of the given validation study.

In-house method validation does typically not address method reproducibility or betweenlaboratory precision. In 2013, four rounds of interlaboratory comparison (ILC) studies were organised by Hole et al. (2013). A total of 12 laboratories participated in the different ILCs. While no measurement instructions were provided to the ILC participants during the first ILC, detailed protocols for sample preparation, video acquisition and data analysis were disseminated to the participants of the next ILC rounds. For a nominal $100 \mathrm{~nm}$ polystyrene reference material, and by using robust statistics, the reproducibility, expressed as a relative standard deviation, could be improved from $40 \%$ (ILC 1) to about $10 \%$ (ILC 2) and 3\% (ILC 3 and ILC 4). The aspect of reproducibility is particularly relevant if data obtained by different laboratories are to be compared. Since the ILC results (Hole et al. 2013) were obtained on a reference material similar to those used during our validation study, it can be assumed that the relative standard deviation of $3 \%$ 
is a reliable estimate of the relative standard uncertainty for method reproducibility. In addition to reproducibility, precision also includes repeatability and intermediate precision. Repeatability indicates the closeness of results performed over a short period, using the same instrument and method, and performed by the same operator. On the other hand, intermediate precision mainly reflects the closeness of results obtained over different sample runs and days.

The relative standard uncertainty for precision $\left(u_{\text {prec }}\right)$ was estimated following Eq. (2). It must be emphasised that for our validated PTA method, the standard uncertainty for precision will only be valid for the mean calculated from the results of three replicates (aliquots) obtained on a single day by one laboratory, which is a typical measurement scheme applied by laboratories, hence the choice of the values for $n_{\text {rep }}, n_{\text {day }}$ and $n_{\text {lab }}$ in the denominators in Eq. (2).

$$
\begin{aligned}
u_{\text {prec }} & =\sqrt{\frac{\mathrm{RSD}_{\mathrm{r}}^{2}}{n_{\text {rep }}}+\frac{\mathrm{RSD}_{\mathrm{ip}}^{2}}{n_{\text {day }}}+\frac{\mathrm{RSD}_{\mathrm{R}}^{2}}{n_{\mathrm{lab}}}} \\
& =\sqrt{\frac{\mathrm{RSD}_{\mathrm{r}}^{2}}{3}+\frac{\mathrm{RSD}_{\mathrm{ip}}^{2}}{1}+\frac{\mathrm{RSD}_{\mathrm{R}}^{2}}{1}}
\end{aligned}
$$

The RSD value for reproducibility $\left(\mathrm{RSD}_{\mathrm{R}}\right)$ is taken from the fourth ILC study that was conducted in frame of the EU FP7 QualityNano project (Hole et al. 2013), and the values for repeatability $\left(\mathrm{RSD}_{\mathrm{r}}\right)$ and intermediate precision $\left(\mathrm{RSD}_{\mathrm{ip}}\right)$ are calculated based on Eqs. (3) and (4):

$\mathrm{RSD}_{\mathrm{r}}=100 \cdot \frac{\sqrt{\mathrm{MSW}}}{y_{\mathrm{m}}}$

$\mathrm{RSD}_{\text {ip }}=100 \cdot \frac{\sqrt{\frac{\mathrm{MSB}-\mathrm{MSW}}{n}}}{y_{\mathrm{m}}}$

where MSW and MSB are the mean of squares within and between measurement days, respectively, calculated using one-way analysis of variance (ANOVA); $n$ is the number of aliquots per day; and $y_{\mathrm{m}}$ is the mean value of the replicate particle size results.
If MSW > MSB, then $\mathrm{RSD}_{\text {ip }}$ cannot be calculated due to the negative argument under the square root. In such situation, the value for $\mathrm{RSD}_{\text {ip }}{ }^{*}$ is calculated alternatively according to Eq. (5) (Federer 1968).

$\mathrm{RSD}_{\mathrm{ip}}^{*}=100 \cdot \frac{\sqrt{\frac{(\mathrm{MSB}-\mathrm{MSW})+\mathrm{MSW}}{n} \cdot e^{-\left(\frac{\mathrm{MSB}}{\mathrm{MSW}}\right)}}}{y_{\mathrm{m}}}$

For each measurand (mode, median and mean), the trueness of the method was quantitatively assessed in terms of experimental bias ( $\left.\Delta_{\text {bias }}\right)$, which is the absolute difference between the certified PTA values of ERMFD101b and the mean values of the replicate measurement results. The assessment, including the estimation of uncertainty for method trueness $\left(u_{\mathrm{t}}\right)$, was performed according to the procedures described in ERM Application Note 1 (Linsinger 2010). The trueness assessment study can be split in three distinct steps.

During the first step, MSB and MSW were calculated for each measurand from the $3 \times 3$ data matrix using one-way ANOVA. The relative standard measurement uncertainty $\left(u_{\text {meas }}\right)$ associated to the mean of the results of the nine replicates was calculated using Eq. (6). The RSD values for repeatability and intermediate precision were again calculated using Eqs. (3)-(4).

$u_{\text {meas }}=\sqrt{\frac{\mathrm{RSD}_{\mathrm{r}}^{2}}{3}+\frac{\mathrm{RSD}_{\mathrm{ip}}^{2}}{3}+\frac{\mathrm{RSD}_{\mathrm{R}}^{2}}{1}}$

At first sight, Eqs. (2) and (6) are almost identical with the only difference being the value of $n_{\text {day }}$. In Eq. (6), the value of 3 for $n_{\text {day }}$ is, however, essential because $u_{\text {meas }}$ must reflect the three measurement days during which the CRM was measured while $u_{\text {prec }}$ will be used for estimating the uncertainty of measurement results on routine samples obtained during only 1 day.

In the second step, the relative standard uncertainty for trueness $\left(u_{\mathrm{t}}\right)$ is estimated by combining the relative standard uncertainty $\left(u_{\mathrm{CRM}}\right)$ of the certified value and the value of $u_{\text {meas }}$ (Eq. 7).

$u_{\mathrm{t}}=\sqrt{u_{\text {meas }}^{2}+u_{\mathrm{CRM}}^{2}}$

In the third and final step, the significance of the experimental bias is evaluated in absolute terms against $u_{\mathrm{t}}$, i.e. if $\Delta_{\text {bias }} \leq 2 \times u_{\mathrm{t}}$, then it can be concluded that the bias is not statistically significant.

As shown in Table 5, no significant biases were obtained for ERM-FD101b results analysed with 
Table 5 Trueness assessment using ERM-FD101b (results in nm)

\begin{tabular}{|c|c|c|c|c|c|c|}
\hline & \multicolumn{3}{|c|}{ NTA 2.3} & \multicolumn{3}{|c|}{ NTA 3.0} \\
\hline & Mode & Median & Mean & Mode & Median & Mean \\
\hline Certified value & 82 & 82 & 87 & 82 & 82 & 87 \\
\hline Standard uncertainty of certified value $\left(u_{\mathrm{CRM}}\right)$ & 2 & 2 & 2 & 2 & 2 & 2 \\
\hline Mean measured value & 82.3 & 83.8 & 87.8 & 87.0 & 77.7 & 84.1 \\
\hline Measurement uncertainty $\left(u_{\text {meas }}\right)$ & 2.7 & 2.7 & 2.8 & 2.6 & 2.6 & 2.7 \\
\hline Trueness uncertainty $\left(u_{\mathrm{t}}\right)$ & 3.4 & 3.3 & 3.5 & 3.3 & 3.3 & 3.4 \\
\hline Absolute bias $\left(\Delta_{\text {bias }}\right)$ & 0.3 & 1.8 & 0.8 & 5.0 & 4.3 & 2.9 \\
\hline Significant? & No & No & No & No & No & No \\
\hline
\end{tabular}

software NTA 2.3 and NTA 3.0. While the biases are not significant, it was observed that the biases for results analysed with NTA 3.0 were significantly higher than those from NTA 2.3. The certified PTA values assigned to ERM-FD101b are based on results from an interlaboratory comparison study (Ramaye et al. 2016) during which laboratories analysed results using both NTA 2.3 and NTA 3.0 software. During that ILC study, results from NTA 2.3 and NTA 3.0 agreed well. As a result, there is no firm indication that the two software versions provide systematically different results.

The expanded measurement uncertainties $(U)$ were finally obtained by combining the individual standard uncertainty contributions from precision and trueness and by multiplying with a coverage factor of $k=2$ (for approximately $95 \%$ confidence interval) according to Eq. (8). Note that the uncertainty related to the lengthscale calibration of the microscope's field of view is considered negligible compared to the uncertainties from trueness and precision and is, therefore, not included in the uncertainty budget.

$U=k \cdot \sqrt{u_{\text {prec }}^{2}+u_{\mathrm{t}}^{2}}$

An overview of the standard and expanded measurement uncertainties for the mode, arithmetic mean and median of the particle number-weighted PSDs is given in Tables 6, 7 and 8 .

When estimating measurement uncertainties from method validation data, the main challenge is often related to the trueness assessment because, in the absence of suitable CRMs, analysts typically use either commercially available non-certified RMs or prepare their own working standards by spiking. The possible risk of applying such approaches is that the true bias cannot be reliably discerned because the measured differences are often a reflection of the contrasting measurement principles of the different methods mostly used for characterisation and/or assigning reference values. In this validation study, we had the advantage of having a fit-for-purpose CRM (ERM-FD101b) available that has been certified using PTA.

As can be seen from the presented uncertainty budgets, the PTA method has a precision in the range of about $3 \%$ to $5 \%$. However, as indicated by the $d_{90}$ values (Table 4), PSDs were sometimes positively skewed due to the presence of agglomerates. The formation of agglomerates can be triggered by the reduced surfactant concentration which is a direct consequence of the high dilution factors required for PTA. In particular for PSL46, of which its nominal particle size approaches the lower LOQ of $41 \mathrm{~nm}$ and where, because of the particle

Table 6 Relative standard and expanded measurement uncertainties (\%) for modal particle size results

\begin{tabular}{|c|c|c|c|c|c|c|}
\hline \multirow[t]{2}{*}{ Uncertainty component } & \multicolumn{3}{|l|}{ NTA 2.3} & \multicolumn{3}{|l|}{ NTA 3.0} \\
\hline & PSL-46 & PSL-100 & ERM-FD102 (size class B) & PSL-46 & PSL-100 & ERM-FD102 (size class B) \\
\hline Precision $\left(u_{\text {prec }}\right)$ & 5.3 & 3.8 & 3.7 & 3.3 & 3.6 & 3.1 \\
\hline Trueness $\left(u_{\mathrm{t}}\right)$ & 4.1 & 4.1 & 4.1 & 4.0 & 4.0 & 4.0 \\
\hline Expanded $(U)$ & 13.2 & 11.2 & 11.0 & 10.3 & 10.8 & 10.2 \\
\hline
\end{tabular}


Table 7 Relative standard and expanded measurement uncertainties (\%) for mean particle size results

\begin{tabular}{|c|c|c|c|c|c|c|}
\hline \multirow[t]{2}{*}{ Uncertainty component } & \multicolumn{3}{|c|}{ NTA 2.3} & \multicolumn{3}{|c|}{ NTA 3.0} \\
\hline & PSL-46 & PSL-100 & ERM-FD102 (size class B) & PSL-46 & PSL-100 & ERM-FD102 (size class B) \\
\hline Precision $\left(u_{\text {prec }}\right)$ & 8.2 & 4.2 & 4.2 & 8.0 & 5.0 & 3.5 \\
\hline Trueness $\left(u_{\mathrm{t}}\right)$ & 4.0 & 4.0 & 4.0 & 3.9 & 3.9 & 3.9 \\
\hline Expanded $(U)$ & 18.2 & 11.6 & 11.6 & 17.8 & 12.7 & 10.6 \\
\hline
\end{tabular}

size its sixth power relationship, the agglomerates scatter light about 35 times more intensely than the smaller primary particles, it is observed that NTA 3.0 offers a much better repeatability $(\sim 3 \%)$ than NTA $2.3(\sim 6 \%)$. This can be attributed to the superior resolution of the FTLA algorithm. Also, a lack of precision is mostly pronounced when evaluating mean particle size results. This is logical as the mean or central gravity value, which is calculated from the entire peak, can be significantly affected by the agglomerates present in the higher percentiles of the distribution. For the tested PSL materials, the small peaks of the agglomerates were only marginally separated from the main peak of the primary particles, and the PTA software only calculated a global mean value rather than a mean value of the primary particles alone. In order to obtain a better precision for the arithmetic mean particle size measurand, one could consider either evaluating the PSDs manually or applying an automatic particle size cut-off value in the video analysis software. Such interventions should, however, be executed with great care as it may also eliminate larger primary particles from the PSD.

Overall, it is safe to state that the validated PTA method can be used to measure the modal and median particle size values of monodisperse suspensions of polystyrene and silica with an accuracy of about $11 \%$ which is only slightly above the pre-defined target of $10 \%$. When approaching the lower LOQ, the measurement uncertainty increases to about $14 \%$. In addition, when using the software NTA 3.0, mixtures of different particle populations can be readily resolved and quantified provided the particle size and concentration of the two individual populations is within the validated working ranges and the ratio of small to big particles is at least 1.25 - that is, sample mixtures in which the modal size of the smallest particles is about $25 \%$ smaller than the modal size of the biggest particles.

\section{Robustness}

As part of the method optimisation process (see ESI), the effect of selected critical method parameters such as the dilution factor, temperature, camera gain and shutter speed was systematically investigated for PSL-100.

Figure $\mathrm{S} 2$ shows that the calculated particle size and the measurement temperature are linearly correlated and that the modal size results evaluated with NTA 2.3 and obtained at a temperature of $24^{\circ} \mathrm{C}$, or higher, agreed best with the size range assigned to PSL-100. For results analysed with NTA 3.0, a good agreement was found for the temperature range of $20{ }^{\circ} \mathrm{C}$ to $27{ }^{\circ} \mathrm{C}$. Temperature control is usually more difficult for measurements performed near ambient conditions than for measurements at a slightly higher temperature. Therefore, it was decided to perform the actual validation measurements in the range of $24{ }^{\circ} \mathrm{C}$ to $26^{\circ} \mathrm{C}$.

A second key parameter that is related to the video recording process is the setting of the camera gain. The camera gain defines the sensitivity of the camera. The calculated particle size value decreased with increasing camera gain (Fig. S3). This trend is most pronounced for results computed with software NTA 2.3. For modal size

Table 8 Relative standard and expanded measurement uncertainties (\%) for median particle size results

\begin{tabular}{|c|c|c|c|c|c|c|}
\hline \multirow[t]{2}{*}{ Uncertainty component } & \multicolumn{3}{|l|}{ NTA 2.3} & \multicolumn{3}{|l|}{ NTA 3.0} \\
\hline & PSL-46 & PSL-100 & ERM-FD102 (size class B) & PSL-46 & PSL-100 & ERM-FD102 (size class B) \\
\hline Precision $\left(u_{\text {prec }}\right)$ & 5.4 & 3.9 & 3.5 & 4.0 & 4.0 & 3.1 \\
\hline Trueness $\left(u_{\mathrm{t}}\right)$ & 4.0 & 4.0 & 4.0 & 4.0 & 4.0 & 4.0 \\
\hline Expanded $(U)$ & 13.6 & 11.2 & 10.8 & 11.4 & 11.3 & 10.2 \\
\hline
\end{tabular}


values analysed with NTA 2.3 and NTA 3.0, a camera gain value up to 250 and 500 yielded acceptable results.

The shutter speed defines the times the camera shutter is open. During the optimisation process, the shutter speed was varied from $12 \mathrm{~ms}$ to $1500 \mathrm{~ms}$ (Fig. S4). No significant effect between particle size and shutter speed range was observed, neither for data analysed with NTA 2.3 nor for data analysed with NTA 3.0.

Other parameters which may have an effect on the measurement result, and which were not considered during the method optimisation stage, were the video capture time and the detection threshold. Both parameters were already studied by Gross et al. (2016) using a monodisperse PSL material with nominal diameter of $600 \mathrm{~nm}$ and bovine serum albumin particles with a size of about $200 \mathrm{~nm}$. The experiments conducted by Gross et al. demonstrated that the modal particle size values that were analysed at different capture times ( $30 \mathrm{~s}$ to $215 \mathrm{~s}$ ) and different detection thresholds (values 5 to 10) did not significantly differ from each other and from the assigned size value.

To ensure the robustness of the validated PTA method, different experimental parameters (Table 9) were deliberately varied during the analysis of PSL-46, PSL-100 and ERM-FD102. All other parameters were either kept constant (e.g. frame rate, bin width of $1 \mathrm{~nm}$ ) or were set to automatic (e.g. blur, maximum jump distance) which then allowed the software programme to determine the optimal conditions for the given sample aliquot. It can be concluded that the validated method is robust against the applied parameter variations and that any potential effect is covered by the repeatability and intermediate precision of the validated method.

\section{Conclusion}

The PTA method has been fully validated for the determination of modal, median and arithmetic mean values of number-weighted PSDs. Various fit-for-purpose silica and polystyrene reference materials consisting of nearspherical and spherical particles were selected, and measurements were carried out according to a nested scheme. This approach allowed, via one-way ANOVA, to analyse the differences between and within measurement days, thereby effectively separating the variances from method repeatability and intermediate precision. In addition to precision, the trueness of the PTA method was assessed by analysing a monodisperse colloidal silica CRM under intermediate precision conditions.
Table 9 Method parameters and parameter values used during the PTA validation study

\begin{tabular}{ll}
\hline Parameter & Level \\
\hline $\begin{array}{l}\text { Sample preparation- } \\
\text { dilution factors }\end{array}$ & \\
PSL-46 & $1 \times 10^{5}, 5 \times 10^{5}$ and $1 \times 10^{6}$ \\
PSL-100 & $1 \times 10^{5}, 5 \times 10^{5}$ and $1 \times 10^{6}$ \\
ERM-FD102 & $1 \times 10^{3}, 1.5 \times 10^{3}$ and $2 \times 10^{3}$ \\
Video acquisition & \\
Camera gain & $250-400$ \\
Camera shutter & 12 ms to 25 ms \\
Frame rate & 25 frames/s \\
Capture time & $60 \mathrm{~s}$ \\
Video analysis & \\
Detection threshold & $4-10$ \\
Blur & Automatic adjustment \\
Minimum expected & Automatic adjustment \\
particle size & Minimum track length
\end{tabular}

The validation study included uncertainty estimation using a simple model where type A relative standard uncertainties for precision and trueness were combined. At the beginning of the study, the target relative expanded uncertainty was set to $10 \%$. For measurements performed in the range of $50 \mathrm{~nm}$ to $100 \mathrm{~nm}$ (not too close to the lower LOQ, i.e. $41 \mathrm{~nm}$ ), this criterion is met since uncertainties vary from $10 \%$ to $11 \%$. Despite its inherent resolving power stemming from the particle-by-particle measurement principle, the PTA method lacks accuracy when determining the arithmetic mean values of particles whose sizes are close to the lower LOQ and PSDs that are skewed due to the presence of agglomerates. The modal and median measurands are found to be equally robust. Measurement uncertainties estimated from the PTA validation data agree well with uncertainties estimated previously by De Temmerman et al. (2014b). However, the PTA measurement uncertainties are a factor two larger than uncertainties for TEM and DLS measurement results (De Temmerman et al. 2014b; Braun et al. 2011).

The results and measurement uncertainties, presented in this contribution, may be used by other laboratories to help them in estimating the uncertainties of their own PTA results. To do so, the laboratory must ensure that their measurements are performed strictly within the scope of the validated method and that the validity of the modified Stokes-Einstein equation is not violated 
Acknowledgments The authors would like to thank Mr. Emmanuel Duh (JRC) for proofreading this manuscript.

\section{Compliance with ethical standards}

Conflict of interest The authors declare that they have no conflict of interest.

Disclaimer Certain commercial equipment, instruments and materials are identified in this paper to specify adequately the experimental procedure. In no case does such identification imply recommendation or endorsement by the European Commission, nor does it imply that the material or equipment is necessarily the best available for the purpose.

Open Access This article is distributed under the terms of the Creative Commons Attribution 4.0 International License (http:// creativecommons.org/licenses/by/4.0/), which permits unrestricted use, distribution, and reproduction in any medium, provided you give appropriate credit to the original author(s) and the source, provide a link to the Creative Commons license, and indicate if changes were made.

\section{References}

Braun A, Couteau O, Franks K, Kestens V, Roebben G, Lamberty A, Linsinger TPJ (2011) Validation of dynamic light scattering and centrifugal liquid sedimentation methods for nanoparticle characterisation. Adv Powder Technol 22:766-770

Contado C (2015) Nanomaterials in consumer products: a challenging analytical problem. Front Chem 3:48

EC (2011) Commission Recommendation of 18 October 2011 on the definition of nanomaterial. (2011/696/EU) OJ L 275, p. 38-40

EURACHEM (2014) The fitness for purpose of analytical methodsa laboratory guide to method validation and related topics, https:/www.eurachem.org/index.php/publications/guides/mv. Accessed 18 Apr 2017

De Temmerman P-J, Verleysen E, Lammertyn J, Mast J (2014a) Size measurement uncertainties of near monodisperse, nearspherical nanoparticles using transmission electron microscopy and particle-tracking analysis. J Nanopart Res 16:2628

De Temmerman P-J, Lammertyn J, De Ketelaere B, Kestens V, Roebben G, Verleysen E, Mast J (2014b) Measurement uncertainties of size, shape, and surface measurements using transmission electron microscopy of near-monodipserse, near-spherical nanoparticles. J Nanopart Res 16:2177

Federer WT (1968) Non-negative estimators for components of variance. J R Stat Soc C 17:171-174

Filipe V, Hawe A, Jiskoot W (2010) Critical evaluation of nanoparticle tracking analysis (NTA) by NanoSight for the measurement of nanoparticles and protein aggregates. Pharm Res 27:796-810

Gallego-Urrea JA, Hammes J, Cornelis G, Hassellöv M (2014) Multimethod 3D characterization of natural plate-like nanoparticles: shape effects on equivalent size measurements. J Nanopart Res 16:2383

Gross J, Sayle S, Karow AR, Bakowsky U, Garidel P (2016) Nanoparticle tracking analysis of particle size and concentration detection in suspensions of polymer and protein sample: influence of experimental and data evaluation parameters. Eur J Pharm Biopharm 104:30-41

Hole P, Sillence K, Hannell C et al (2013) Interlaboratory comparison of size measurements on nanoparticles using nanoparticle tracking analysis (NTA). J Nanopart Res 15:2101

ISO/IEC Guide 98-3 (2008) Uncertainty of measurement - part 3: guide to the expression of uncertainty in measurement (GUM: 1995). International Organization for Standardization, Geneva

Kestens V, Roebben G, Herrmann J et al (2016) Challenges in the size analysis of a silica nanoparticle mixture as candidate certified reference material. J Nanopart Res 18:171

Krueger AB, Carnell P, Carpenter F (2016) Characterization of factors affecting nanoparticle tracking analysis results with synthetic and protein nanoparticles. J Pharm Sci 105:1434-1443

Linsinger TPJ (2010) Comparison of a measurement result with the certified value. ERM Application Note 1. http://www. erm-crm.org. Accessed 18 Apr 2017

Malloy A, Carr B (2006) Nanoparticle tracking analysis - the Halo $^{\text {TM }}$ system. Part Part Syst Charact 23:197-204

McDonald SA, Daniels CA, Davidson JA (1977) Determination of the true size of poly(vinyl chloride) lattices by electron microscopy using a vertical shadowing technique. J Colloid Interface Sci 59:342-349

Nicolet A, Meli F, van der Pol E (2016) Inter-laboratory comparison on the size and stability of monodisperse and bimodal synthetic reference particles for standardization of extracellular vesicle measurements. Meas Sci Technol 27:035701

Patois E, Capelle MAH, Palais C, Gurny R, Arvinte T (2012) Evaluation of nanoparticle tracking analysis (NTA) in the characterization of therapeutic antibodies and seasonal influenza vaccines: pros and cons. J Drug Del Sci Technol 22: $427-433$

Peters RJB, Bouwmeester H, Gottardo S et al (2016) Nanomaterials for products and application in agriculture, feed and food. Trends Food Sci Technol 54:155-164

Qian H, Sheetz MP, Elson EL (1991) Single particle trackinganalysis of diffusion and flow in two-dimensional systems. Biophys J 60:910-921

Ramaye Y, Kestens V, Braun A, Linsinger T, Roebben G (2016) The certification of equivalent diameters of silica nanoparticles in aqueous solution: ERM-FD101b. Certification report. Publications Office of the European Union, Luxembourg

Sauvain J-J, Suarez G, Edmé J-L, Bezerra OMPA, Silveira KGS, Amaral LS, Carneiro APS, Chérot-Kornobis N, Sobaszek A, Hulo S (2017) Method validation of nanoparticle tracking analysis to measure pulmonary nanoparticle content: the size distribution in exhaled breath condensate depends on occupational exposure. J Breath Res 11:016010

Tsai D-H, DelRio FW, Keene AM, Tyner KM, MacCuspie RI, Cho TJ, Zachariah MR, Hackley VA (2011) Adsorption and conformation of serum albumin protein on gold nanoparticles investigated using dimensional measurements and in situ spectroscopic methods. Langmuir 27:2464-2477

Tuoriniemi J, Johnsson A-C JH, Holmberg JP, Gustafsson S, Gallego-Urrea JA, Olsson E, Pettersson JBC, Hassellöv M (2014) Intermethod comparison of the particle size distributions of colloidal silica nanoparticles. Sci Technol Adv Mater 15:035009

Walker JG (2012) Improved nano-particle tracking. Meas Sci Technol 23:065605 